Research Article

\title{
Computational Analysis of Fluid Flow through a Sine-Curved Channel with High Reynolds Number
}

\author{
Abid A. Memon, ${ }^{1}$ M. Asif Memon ${ }^{1},{ }^{1}$ Kaleemullah Bhatti, ${ }^{1}$ Hammad Alotaibi, ${ }^{2}$ \\ Y.S. Hamed $(1),{ }^{2}$ Gul M. Shaikh, ${ }^{1}$ and Ilyas Khan $\left(^{3}{ }^{3}\right.$ \\ ${ }^{1}$ Department of Mathematics, Sukkur IBA University, Sukkur 65200, Sindh, Pakistan \\ ${ }^{2}$ Department of Mathematics and Statistics, College of Science, Taif University, P. O. Box 11099, Taif 21944, Saudi Arabia \\ ${ }^{3}$ Department of Mathematics, College of Science Al-Zulfi, Majmaah University, Al-Majmaah, P.O. Box 66, \\ Majmaah 11952, Saudi Arabia
}

Correspondence should be addressed to Ilyas Khan; i.said@mu.edu.sa

Received 13 February 2021; Revised 29 March 2021; Accepted 8 October 2021; Published 21 October 2021

Academic Editor: Taha Aziz

Copyright (c) 2021 Abid A. Memon et al. This is an open access article distributed under the Creative Commons Attribution License, which permits unrestricted use, distribution, and reproduction in any medium, provided the original work is properly cited.

\begin{abstract}
In this paper, we attend to investigate the steady flow of a Newtonian fluid through a sine-curved channel working with the leastsquare technique of Galerkin's approach. We implement the whole simulation using Comsol Multiphysics 5.4. To study the fluid flow through this channel, we take the Reynolds numbers in the range from 1000 to 10,000 and amplitude of the sine-curved channel in the range from $10 \mathrm{~cm}$ to $30 \mathrm{~cm}$. We examine the flow rate and pressure at the outlet. It is observed that, at the outlet, maximum speed is increasing linearly along the Reynolds number and that the maximum pressure settled a negative relationship with the Reynolds number when increased. It is also determined that due to an increase in the hydraulic jumps, when increasing the amplitude of vibration of the channel, the velocity of flow got fluctuated at the above walls, which also results in a decline in the pressure from the inlet to exit of the channel. Moreover, the several correlations keeping amplitude as constant have been developed for the maximum flow velocity magnitude at the exit of the channel relating to the Reynolds number. These correlations will be definitely used for the future production and comparison for the fluid flow for the curvy channel.
\end{abstract}

\section{Introduction}

The perceptions of the fluid flow occasions are happening everywhere in the universe [1-3], for example, in engineering and modern applications [4-6]. The streams of the basic models are flying creatures [1], stalagmites production in the sea [2], falling motions of hailstones [3], spreading of contamination [7], the instances of penetrating in the petroleum engineering [8], movement of planes in the sky [9], atomic explosions in the field of nuclear energy [10], heat reduction of solar-powered batteries in the field of energy sciences [11], creation of a fine exchanger [12], and so on. Fluid flow through the pipes with curvy shapes in the field of computational fluid dynamics is under investigation by several authors for decades due to its application in the industry and research. Fluid flow through the pipes with curvy shapes is considerably complicated as compared to the flow through pipes or rectangular slits. The transportation of urine organized between the bladder and kidney, a stream of the chime in the direction of the small and large intestine, the unforeseen motion of the blood vessels, and crusade of caustic fluid are few applications. Velocity of the fluid and pressure distribution within the fluid depend upon the shape of the channel, and therefore, several scientists and researchers set their light of intellect upon the current topic of fluid flow through the curved channels to talk about the various parameters used in industrial applications.

An observation and examination [13] was conducted for the fluid flow along with the heat transfer through a sinusoidal channel by considering a fully developed flow at the inlet taking the wavelength of the channel to be $0.04 \mathrm{~m}$. It was discovered that, with the rise in the Reynolds number, the pressure drops significantly. This phenomenon is in contrast with the flow through rectangular channels. The 
fluid flow along with heat transfer through the two-dimensional sinusoidal wavy channel is inspected in [14] with the help of a simple algorithm. The fluid is fully developed and the range of the Reynolds number is from 100 to 1000 . It was observed that the increase in the Reynolds number also increases the isotherms in the curved wall as well as the heat transfer rate. Several experiments [15] were performed on the curvy geometry considering the single-phase, Newtonian, laminar, and turbulent fluid flows with the Reynolds numbers in the range $0<\operatorname{Re}<7500$. It was determined that due to the boost in the flow rate of the curvy channel as compared to the rectangular regions, the heat transfer is increasing significantly. Escorted by the help of the powerlaw model of non-Newtonian fluid, a peristaltic flow through the curvy channel was examined in [16]. A third-grade fluid was used to obtain that, with the increase in the wavelength of the vibration of the channel, pressure is decreased. Decomposing the governing equations [17] in the harmonic one with the help of the stream functions of the components of the velocity field, Stokes flow via the sinusoidal channel was determined. It was found that the velocity field, which occurred in the domain, is the function of the amplitude of the oscillation of the wall. Tracking the pattern of fluid flow through the wire gauze or screen, an asymptotic solution that connects the stream-velocity gained through numerical solution compared to the analytic solution was provided in [18] while experiencing air as a working fluid through the rectangular duct connected by parallel plates. On implementing the finite element analysis based on COMSOL Multiphysics 5.4 [19] the emerging technology of leastsquare Galerkin's scheme was implemented, which experienced the fluid flow simulation through the rectangular channel with three screens situated at the middle of the channel with angles in the range from $-45^{\circ}$ to $45^{\circ}$ degrees. They have obtained that the maximum velocity and maximum pressure in the rectangular domain can be further optimized by improving angles. With the implementation of Galerkin's least-square scheme using the COMSOL Multiphysics 5.4 [20], an air laminar flow was examined through the channel fixed with the three screens situated at inclinations from -45 to 45 degree. It was found that, with the increase in resistance coefficients, the optimum velocity in the domain can further be optimized. In the problem that the speed, pressure factor, and temperature are the entropy variables in any flow streams, an overall solution can be framed to address the compressible and incompressible flow issues. The formulation [21] is known as Galerkin's leastsquare scheme and developed by Hauke and Hughes and serving from two decades to solve the complex fluid flow through different geometries. The velocity and the pressure pattern along the circular object was studied [22] with the help of an emerging simulation tool COMSOL Multiphysics 5.4 with the least-square approach. It was stated that the numerical approach is in good agreement with the asymptotic solution for the screen boundary condition. Using the FEM technique with the least-square approach, a laminar flow was studied [23] through the backward steps ranging the Reynolds number 100-1000. The vortex length obtained in the downstream was in good agreement with the experimental results. Moreover, the horizontal and vertical flow patterns were discussed in detail. Several linear regression lines [24] for optimum pressure and velocity in the rectangular domain and the drag force due to screen for in terms of the Reynolds number were expressed using the FEM approach with Galerkin's scheme. With the implementation of the screen boundary condition, another benchmark problem [25] for Newtonian and the nonNewtonian fluid using the power-law model of Oswald-de Waele was observed in the rectangular channel. Several empirical equations relating to optimum velocity as well as pressure with the Reynolds number are found on the basis of power-law indexes used.

\section{Methodology}

2.1. Geometrical Structure and Meshing. The simplified diagram of the sine-curved channel is demonstrated through Figure 1. The length of the channel is dragged to $2 \pi$. The lower boundary of the channel is $A \sin 3 t$, and the upper boundary is $A \sin 3 t+1$, where $A$ is the amplitude of vibration of the channel and $t$ is a parameter with range $-\pi<t<\pi$. The left vertical wall is the inlet, whereas the right vertical wall is recognized as the outlet. An average velocity related with the Reynolds number is tricked on the inlet of the channel to follow the flow right through the domain. The lower and upper frontiers are walls and imposed by the slip condition.

The designated domain is parted into small queer triangular elements displayed in Figure 2. Two thousand eight hundred and eighty (2880) elements were utilized with the mesh area 6.83. To enhance the precision, the minimum quality element is taken 0.5994 with 0.902 the average quality element.

2.2. Governing Incompressible Equations. It has been many years that the Navier-Stokes PDEs are serving to comprehend the fluid course through numerous mind-boggling channels. In this paper, two-dimensional Navier-Stokes equations are worked out by means of a numerical programming tool COMSOL Multiphysics 5.4. Let $V=\left(u_{x}, u_{y}\right)$ be the velocity field with $u$ as the horizontal component and $v$ as the vertical component. We consider the following set of equations:

$$
\begin{aligned}
\frac{\partial \vec{V}}{\partial t}+(\vec{V} \cdot \nabla) \vec{V}+\frac{1}{\rho} \nabla p+\mu \nabla^{2} \vec{V} & =F, \\
\nabla \cdot \vec{V} & =0 .
\end{aligned}
$$

Assumption of the steady state situation implies that

$$
\frac{\partial \vec{V}}{\partial t}=0 .
$$

Fluid flow of the air through the sine-curved channel is going to be analyzed with the nondimensional Reynolds number; therefore, an average velocity profile related with the Reynolds number is imposed at the entrance of the 


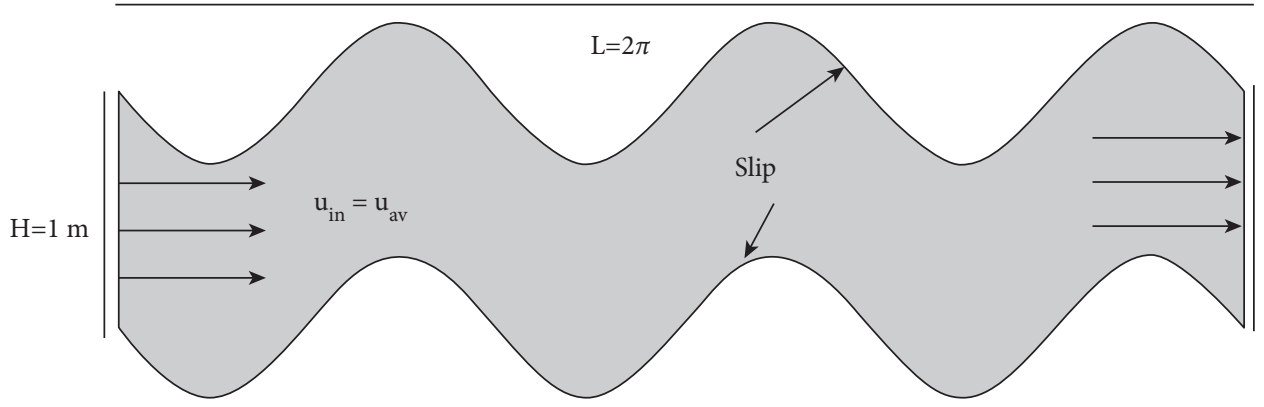

FIgURE 1: Schematic diagram of the channel.

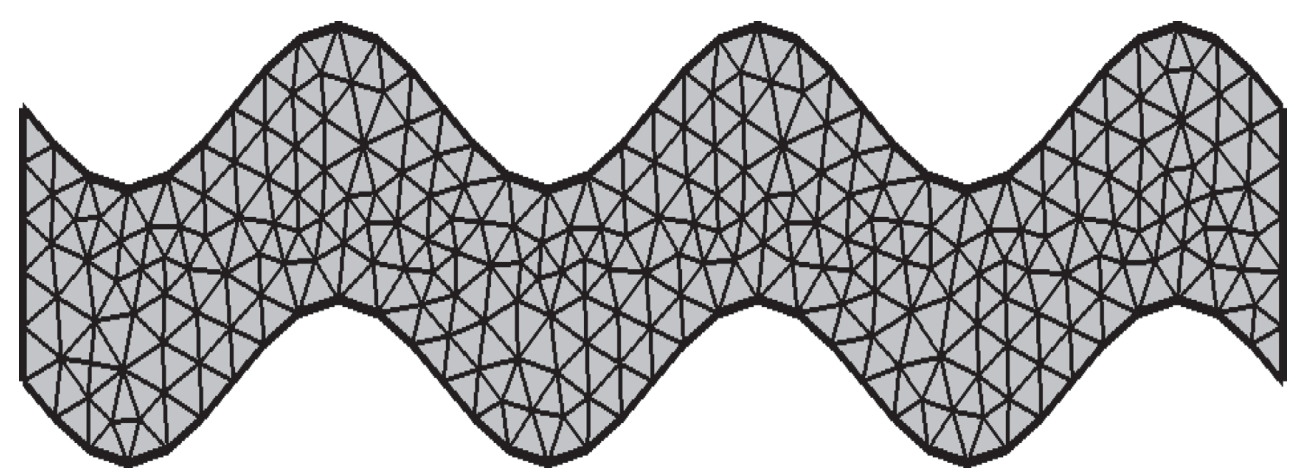

Figure 2: Coarse mesh of the geometry.

channel. Let $\rho, \mu$, and $L$ be the density, viscosity, and length of the channel, respectively; then, the Reynolds number is defined as

$$
\begin{aligned}
& R_{e}=\frac{\text { Inertial force }}{\text { Viscous force }}, \\
& R_{e}=\frac{\rho \mu_{\mathrm{in}} L}{\mu} .
\end{aligned}
$$

To revoke the viscous consequences along the boundaries of the selected channel, we made addition slip-walls on the upper and lower boundaries. If $\vec{K}$ is the operator defined as follows, then we have the following slip boundary conditions:

$$
\begin{array}{r}
\vec{V} \cdot \vec{n}=0, \\
\vec{K}-(\vec{K}-\vec{n}) \vec{n}=0,
\end{array}
$$

where

$$
\vec{K}=v\left(\nabla \cdot \vec{V}+(\nabla \cdot \vec{V})^{T}\right) \vec{n} .
$$

After getting the outcomes for the velocity field magnitude for the domain and dynamic pressure on the extreme wall, it was found that the rate of flow at the outlet has a direct relationship with Re and with the amplitude of the vibration of the channel. Knowing this, we would apply the liner regression process to determine our empirical equations which can be used for future forecast and to test the flow with different numerical methods. The linear equation for any $x$ related to $y$ can be written as

$$
y=A+B x,
$$

where $A$ and $B$ can be determined statistically as

$$
\begin{aligned}
& A=\frac{\sum\left(x_{i}-\bar{x}\right)\left(y_{i}-\bar{y}\right)}{\sum\left(x_{i}-\bar{x}\right)^{2}}, \\
& B=\bar{y}-A \bar{x} .
\end{aligned}
$$

The system of governing equations will be carried through numerical approach of the finite-element method with Galerkin's least-square scheme [9] via commercial software COMSOL Multiphysics.

2.3. Least-Square Scheme. The procedure of the least-square scheme of the finite-element method is only applicable for the first-order partial differential equations. The NavierStokes equations which are the combination of the momentum equation and continuity equation are of $2^{\text {nd }}$ order. Therefore, to employ the current method, they would be linearized by putting the auxiliary equation known from the contents of the fluid dynamics.

Let us consider the boundary value problem of the firstorder partial differential equation with linear operators $L$ and $B$ as the boundary operator.

$$
\begin{aligned}
& \mathrm{Lu}=f \text { in } \Omega \varepsilon R^{2}, \\
& \mathrm{Bu}=g \text { in } \Gamma \varepsilon \Omega .
\end{aligned}
$$

Let us consider the solution vector $u=$ $\left[\begin{array}{lllll}u_{1} & u_{2} & u_{3} & \ldots & u_{m}\end{array}\right]^{T}$ which contains $m$-unknowns. If $A_{i}$ 
and $A$ are the positive definite matrices involving the functions of $x$ as their entries, then equation (12) can be written as

$$
\mathrm{Lu}=\sum_{i=1}^{n_{d}} A_{i} \frac{\partial \mathbf{u}}{\partial x_{i}}+A \mathbf{u} .
$$

The current problem of the fluid flow is examined for the two-dimension only. So, let us involve the set $L^{2}(\Omega)$ that contains the function whose squares are defined on the domain $\Omega$. The inner product in this set defined as follows:

$$
(u, v)=\int_{\Omega} u v \mathrm{~d} \Omega u, \quad v \varepsilon L^{2}(\Omega)
$$

where the norm for the self-inner product is defined as

$$
\|u\|_{0}^{2}=(u, u) .
$$

Let us define a set of Sobolev space

$$
H^{1}(\Omega)=\left\{u \varepsilon L^{2}(\Omega) ; \partial^{\alpha} u \varepsilon L^{2}(\Omega), \quad \forall|\alpha| \leq 1\right\},
$$

where

$$
\alpha=\left\{\alpha_{1}, \alpha_{2}, \alpha_{3}, \ldots, \alpha_{n_{d}}\right\} \varepsilon N^{n_{d}},|\alpha|=\alpha_{1}+\alpha_{2}+\alpha_{3}+\cdots+\alpha_{n_{d}},
$$

which will be redefined as the definition of the norm on the Sobolev space $H^{1}(\Omega)$ as follows.

Also, their norm is

$$
\|u\|_{1}^{2}=\sum_{|\alpha| \leq 1}\left\|\partial^{\alpha} u\right\|_{0}^{2}
$$

where the element $u$ is the vector of $m$-components whose set of product space will be defined as follows:

$$
\begin{aligned}
L^{2}(\Omega) & =\left(L^{2}(\Omega)\right)^{m}, \\
H^{1}(\Omega) & =\left(H^{1}(\Omega)\right)^{m} .
\end{aligned}
$$

For the abovementioned product space, we would like to define the following norms:

$$
\begin{aligned}
& \|u\|_{1}^{2}=\sum_{|\alpha| \leq 1}\left\|\partial^{\alpha} u\right\|_{0}^{2}, \\
& \|\underline{u}\|_{0}^{2}=\sum_{j=1}^{m}\left\|u_{j}\right\|_{0}^{2} .
\end{aligned}
$$

The current problem of the fluid flow is the boundary value problem; therefore, we are also going to explain space sustaining the following conditions on any type of domain:

$$
\underline{S}=\left\{\underline{u} \varepsilon\left(H^{1}(\Omega)\right)^{m} ; B \underline{u}=0 \text { on } \Gamma\right\} .
$$

Let $\underline{u}$ be an arbitrary trial function belonging to $\underline{S}$, and we are considering $f \in \underline{L}^{2}$ and $L: \underline{S} \longrightarrow \underline{L}^{2}$. Now, we are in a position to assume the least-square formulation which is nothing but minimizing the $I(\underline{u})$ which is the inner product of the residuals obtained from the governing set of partial differential equations defined as

$$
I(\underline{u})=\|L \underline{u}-\underline{f}\|_{0}^{2}=(L \underline{u}-\underline{f}, L \underline{u}-\underline{f}) .
$$

From the method of variation of the finite-element method, we have to minimize the energy term by $\delta=0$ and $\delta \underline{u}=\underline{w}$. Later, to get the vector $\underline{u}$, we will use the following formulation:

$$
(L \underline{w}, L \underline{u})=(L \underline{w}, \underline{f}), \quad \forall \underline{w} \in \underline{S} .
$$

Let us divide the whole domain of interest and $\mathrm{Nd}$ denote the nodal values in the discretized domain, and $\Psi_{j}$ is the linear shape function defined on each subdomain. Let us apply the well-known procedure of the finite-element scheme; now, we define

$$
\underline{u}_{h}(\underline{x})=\sum_{j=1}^{N_{e}} \chi_{j}(\underline{x})\left(\begin{array}{c}
u_{1} \\
u_{2} \\
\vdots \\
u_{m}
\end{array}\right)_{j}
$$

where the vector $\left(u_{1}, u_{2}, u_{3}, \ldots, u_{m}\right)_{j}$ is the values on each node for the $j$ th element and $h$ is the length of the element.

Let $U$ be the global solution in the vector form of all nodal values and $K$ be the global matrix that is assembled with the help of local matrices for each element. If $F$ defines the vector gained through the vector elements, then we define the finite-element formulation as

$$
\mathrm{KU}=f,
$$

where

$$
\begin{aligned}
K_{e}= & \int_{\Omega_{d}}\left(L \chi_{1}, L \chi_{2}, L \chi_{3}, \ldots, L \chi_{N e}\right)^{T} \\
& \left(L \chi_{1}, L \chi_{2}, L \chi_{3}, \ldots, L \chi_{N e}\right) d \Omega, \\
F_{e}= & \int_{\Omega_{e}}\left(L \chi_{1}, L \chi_{2}, L \chi_{3}, \ldots, L \chi_{N e}\right)^{T} \underline{f} \mathrm{~d} \Omega,
\end{aligned}
$$

where $\Omega_{e}$ shows the subdomain of the eth element and $T$ shows the transpose, and we define the operator

$$
L \chi_{j}=\chi_{j, x} A_{1}+\chi_{j, y} A_{2}+\chi_{j, z} A_{3}+\chi_{j} A .
$$

The least-square procedure will be easily solved once you get the matrices $A, A_{1}, A_{2}$, and $A_{3}$ from the governing partial differential equation, and $K$ must be the positive definite matrix. The process leads to giving the set of algebraic equations that will be solved later by any method such as the Newton-Raphson procedure.

After implementation of the corresponding scheme of Galerkin's least-square technique, the error estimation summary is given by Table 1 for the last iteration for each Reynolds number.

\section{Validation and Comparison}

Before discussion on the results, we validate by comparing our numerical solution with the analytical solution available in the literature. Earlier, the solution of the fluid flow via the 
TABLE 1: Error estimation for each Re.

\begin{tabular}{lcc}
\hline $\mathrm{Re}$ & Relative error estimate in solution & Relative error estimate in residual \\
\hline 1000 & $2.3 E-15$ & $1.4 E-15$ \\
2000 & $3.8 E-15$ & $2.1 E-15$ \\
3000 & $5.9 E-15$ & $2.2 E-15$ \\
4000 & $2.2 E-15$ & $2.5 E-15$ \\
5000 & $3.6 E-15$ & $2 E-15$ \\
6000 & $3.9 E-15$ & $2.5 E-15$ \\
7000 & $1.1 E-14$ & $2.8 E-15$ \\
8000 & $3.2 E-15$ & $2.9 E-15$ \\
9000 & $4.2 E-15$ & $3.4 E-15$ \\
10,000 & $2.9 E-15$ & $3.2 E-15$ \\
\hline
\end{tabular}

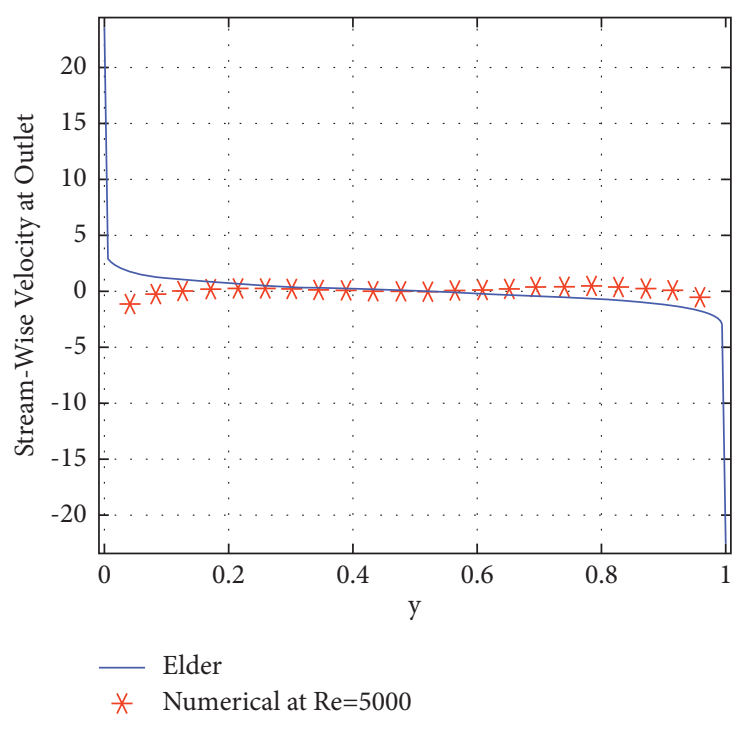

(a)

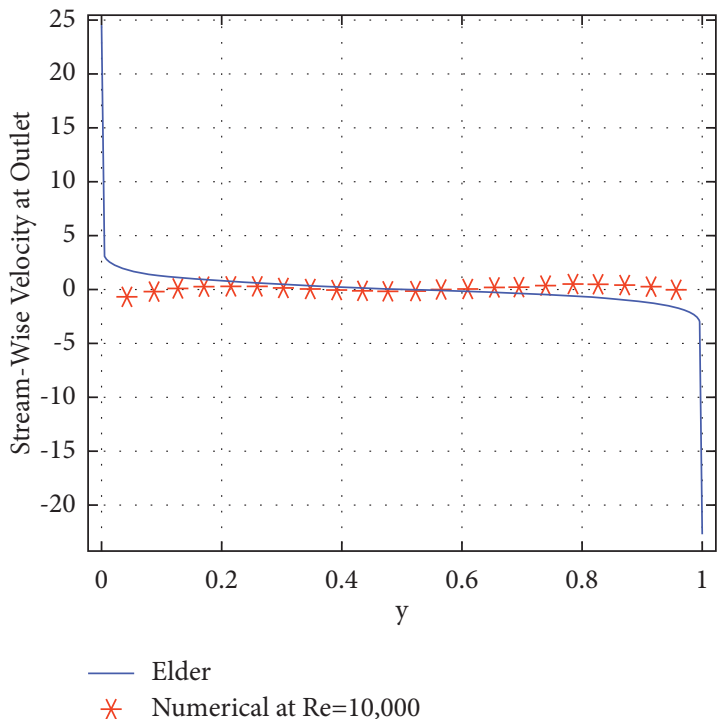

(b)

FIgURE 3: Comparison of the numerical solution with the analytical solution. (a) $\operatorname{Re}=5000$ and (b) $\operatorname{Re} 10,000$.

screens was determined by Elder in 1959, and he developed the equation for the exit of the channel with a small angle that can be used to find the streamwise velocity.

In equation (31), $\mu$ is estimated numerically using other parameters. Both the left-hand and right-hand side of equation (31) are called streamwise velocity. In this problem, we have also tested the same equation by calculating the streamwise velocity at the orifice of the channel by fitting the screen at $45^{\circ}$ degree at the mid of the channel. Figure 3 shows that we have achieved excellent results.

$$
\frac{\left(\mu / \mu_{\text {in }}-1\right)\left(1+\eta+k \cos ^{2} \theta\right)}{(1-\eta) \tan \theta k \cos ^{2} \theta}=\frac{2}{\pi} \log \left(\cot \left(\frac{\pi y}{2}\right)\right)
$$

\section{Results and Discussion}

Numerically calculated results obtained here are presented via surface and graphs for speed and the absolute pressure throughout the domain for different Reynolds numbers and the optimum speed and pressure at the outlet. Finally, we will submit our empirical equations calculated by the linear regression process which show the relation of maximum speed at the outlet of the channel with the Reynolds number as well as with the amplitude of the vibration of the channel.

4.1. Velocity Field. The fully developed flow has been created by allowing air fluid in the channel with the average velocity $\mathrm{u}_{\mathrm{in}}$. In Figure 4 , we present the magnitude of velocity at every point of the channel. It is shown that the distribution of the velocity is not uniform because of the periodic change in the boundaries of the geometry or there might be involvement of the zigzag walls. Due to the hydraulic jumps when the channel slides above, the maximum speed of the fluid always can be seen at the top boundary of the channel and minimum speed at the lower walls. On observing the fluid flow at the inlet and outlet in the channel, the velocity of the fluid is increasing, and also, with an increase in amplitude, the hydraulic jumps will become more powerful to create the maximum velocity at the upper boundary of the channel.

The maximum speed at the outlet of the channel for a certain Reynolds number possesses a linear relationship with the amplitude of vibration, see Figure 5(a). The maximum speed can be seen increasing with the amplitude with the same rate up to $25 \mathrm{~cm}$ of amplitude and then increasing with 


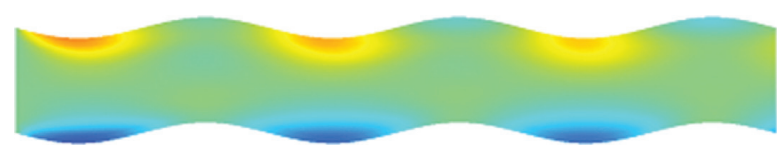

(a)

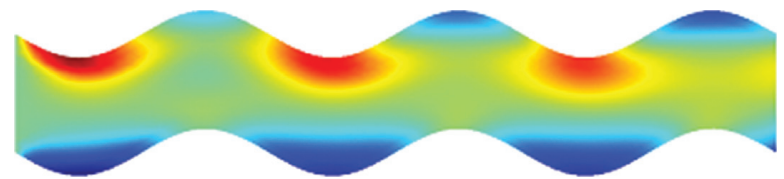

(c)

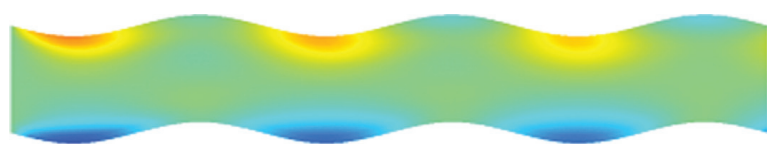

(b)

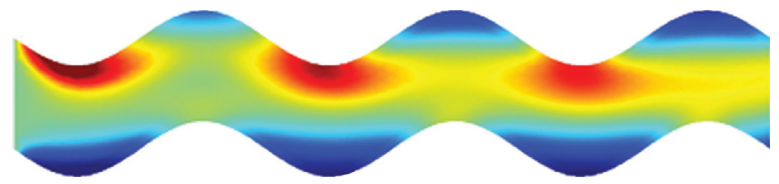

(d)

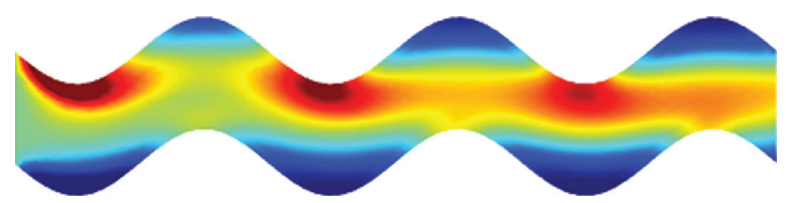

(e)

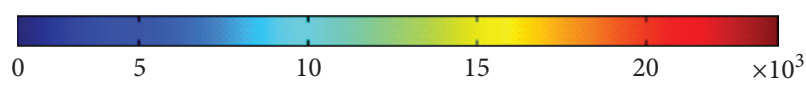

FIgURE 4: Surface plot of the velocity field through the curved channel at $\operatorname{Re}=5000$.

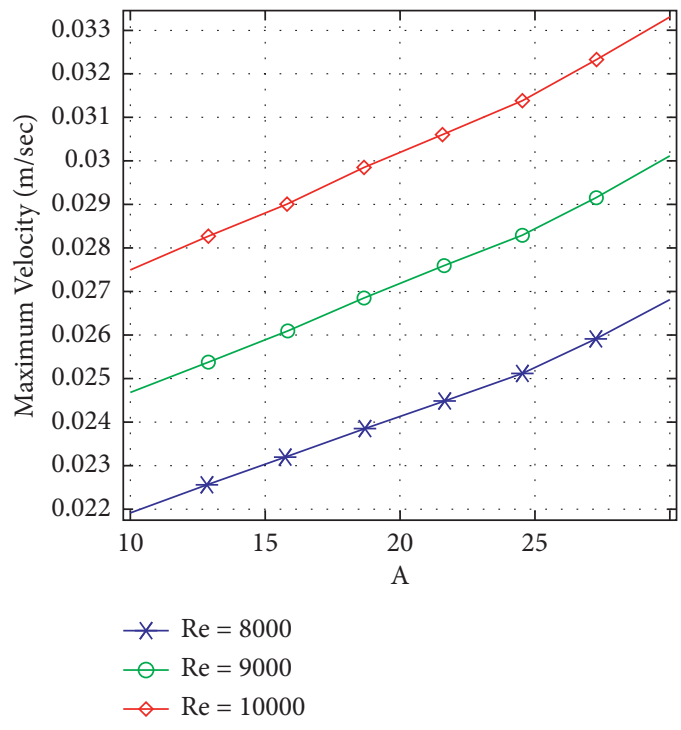

(a)

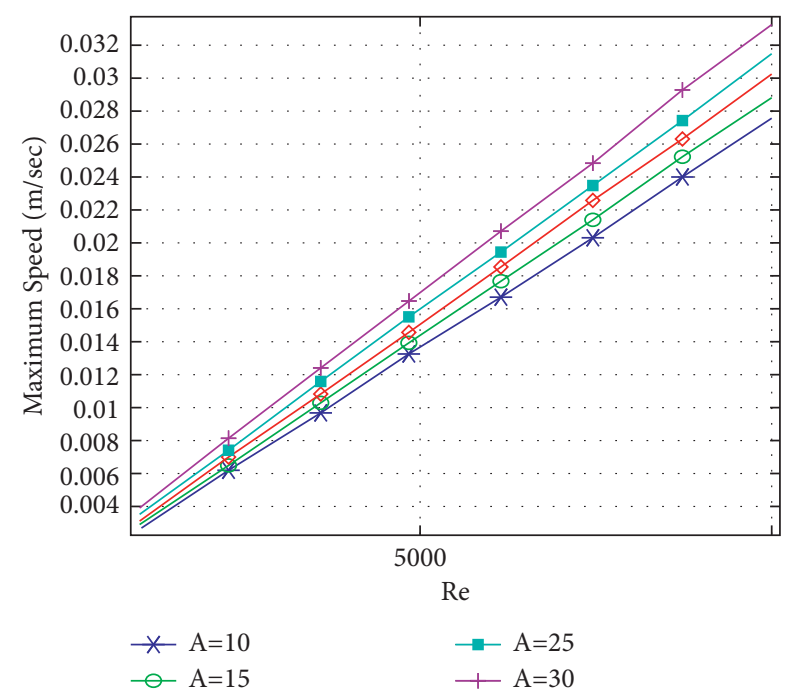

(b)

Figure 5: (a) Maximum flow of the fluid at the exits of the channel against amplitude. (b) Maximum flow of the fluid at the outlet against the Reynolds number.

a different rate after $25 \mathrm{~cm}$. By fixing the amplitude, the maximum speed is calculated at the outlet with increasing Reynolds number, see Figure 5(b). It can be seen that the maximum speed increases by increasing amplitude for all Reynolds numbers due to the hydraulic jumps. In Table 2, we present the regression equations for the maximum speed related with the Reynolds number and with the amplitude.

4.2. Pressure. The pressure distribution is represented through the surface plots in Figure 6 which describe the pressure driven throughout the domain for $\operatorname{Re}=10,000$. It is shown that the dynamic pressure is increasing with the increase in amplitude for the fixed Reynolds number from the
TABLE 2: Regression equations of the maximum velocity and the Reynolds number by fixing amplitudes.

\begin{tabular}{lc}
\hline Amplitude & Equations \\
\hline 10 & $V_{\max }=2.75 E-6 * \mathrm{Re}-0.00012$ \\
15 & $V_{\max }=2.88 E-6 * \mathrm{Re}-0.0004$ \\
20 & $V_{\max }=3 E-6 * \mathrm{Re}+0.00009$ \\
25 & $V_{\max }=3.10 E-6 * \mathrm{Re}+0.00041$ \\
30 & $V_{\max }=3.27 E-6 * \mathrm{Re}+0.00006$ \\
\hline
\end{tabular}

inlet to the outlet of the channel. But, for a fixed amplitude of the vibration, the pressure is decreasing from the entrance to the exit because of hydraulic jumps which results in an increase in the speed of the fluid and decrease in pressure. 


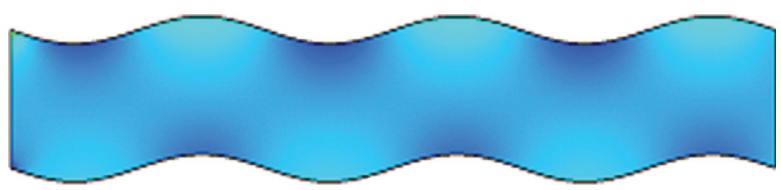

(a)

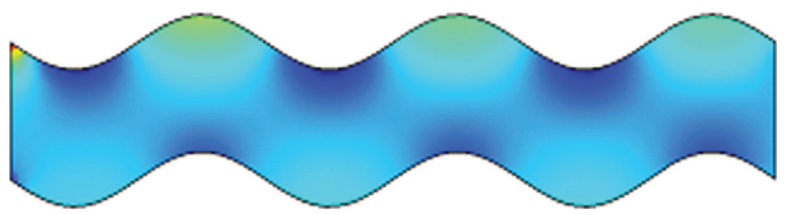

(c)

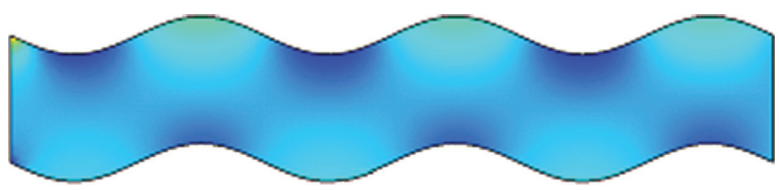

(b)

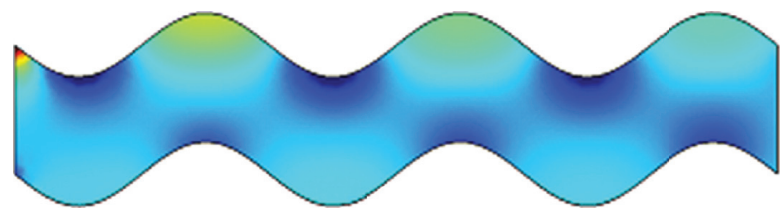

(d)

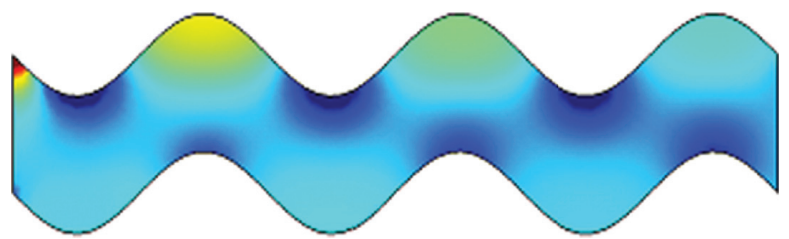

(e)

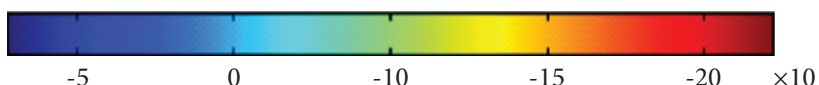

Figure 6: Surface plot of the pressure through the curved channel at $\operatorname{Re}=10,000$.

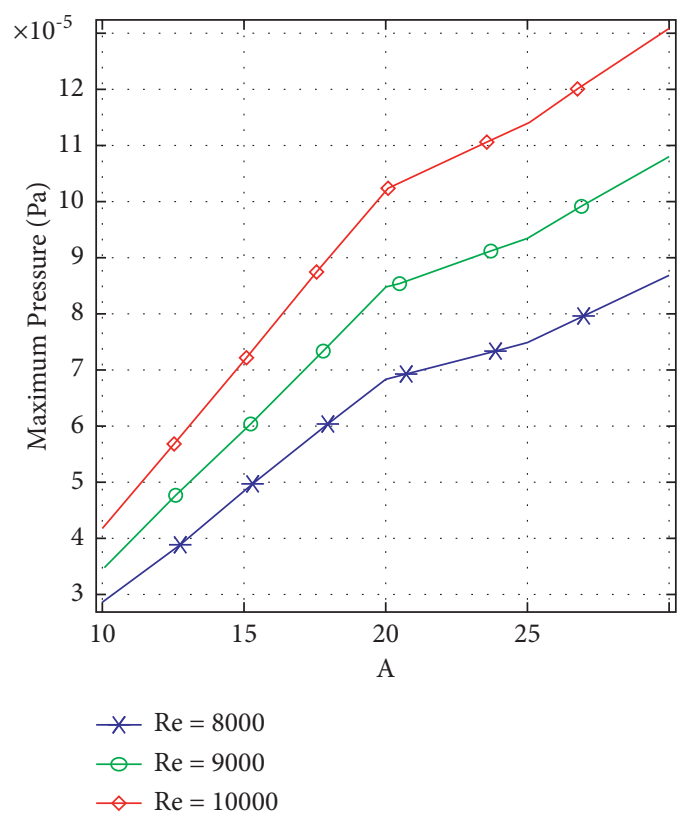

(a)

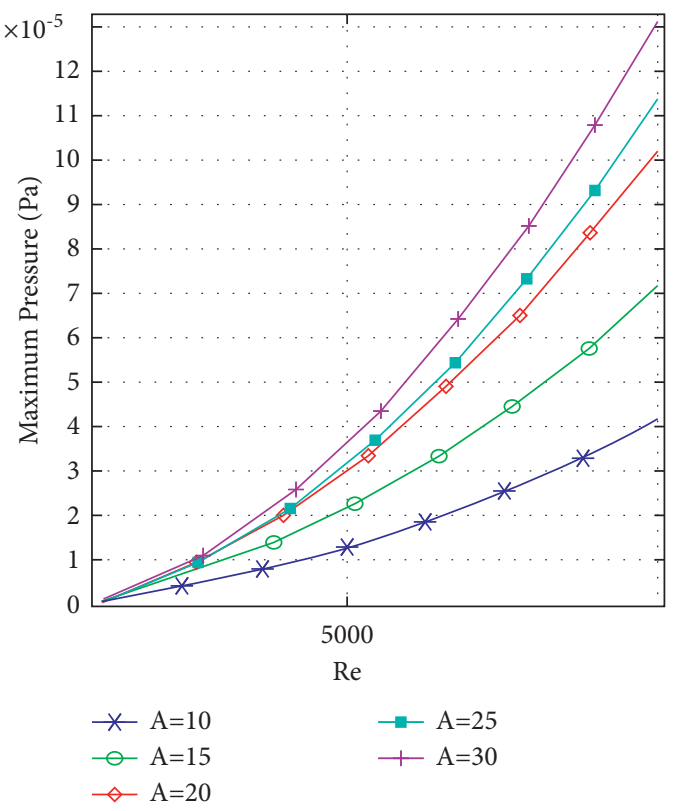

(b)

FIGURE 7: (a) Maximum pressure at the outlet against all amplitudes of vibration. (b) Maximum pressure at the outlet against all Reynolds numbers.

Figure 7(a) describes the maximum pressure calculated at the outlet for $\operatorname{Re}=8000,9000$, and 10000 . For a fixed Reynolds number, the pressure is increasing linearly with the maximum pressure up to $20 \mathrm{~cm}$ of the amplitude of vibration after which it is increasing nonlinearly. Figure 7(b) describes the maximum pressure at the outlet for a fixed amplitude of vibration with varying Reynolds number. This graph indicates that the maximum pressure at the outlet possesses nonlinear, in specific, parabolic relationships with the Reynolds number for a fixed amplitude of vibration of the channel.

\section{Conclusions}

Laminar and Newtonian flow of air through the sine-curved channel with the amplitude of vibration from $10 \mathrm{~cm}$ to $30 \mathrm{~cm}$ 
is analyzed using the finite-element method. Flow patterns are tested with different Reynolds numbers ranging from 1000 to 10,000 . The magnitude of velocity and pressure has been examined for the flow stream, and the obtained results are presented through various plots. The results are compared with the analytic solution with the available literature. We have shown that our solution has a good agreement with the analytical solution, as was expected.

It is shown that, for a fixed amplitude of vibration of the channel, the flow velocity is increasing because of the hydraulic jumps. Through graphs, we have shown that the area where maximum air flow appears is near the upper wall and the area where minimum air flow appears is near the lower wall. Air flow at the outlet of the channel becomes speedy by increasing the Reynolds number, which is natural, but speed also highly depends upon the amplitude of vibration of the channel. Dynamic pressure has also been described by various graphs. It is obtained that, for a fixed Reynolds number, pressure is decreasing from the entrance to exit of the channel with an increase in amplitude. The portions where pressure is minimum appear at the upper boundary. The optimum pressure at the exit elaborates the nonlinear relationship of the pressure with amplitude.

\section{Data Availability}

No data were used to conduct this research.

\section{Conflicts of Interest}

The authors declare no conflicts of interest regarding the publication of this paper.

\section{Acknowledgments}

This research was supported by the Taif University Researchers, Taif University, Supporting Project No. TURSP2020/304, Taif, Saudi Arabia.

\section{References}

[1] D. Floryan, T. Van Buren, and A. J. Smits, "Efficient cruising for swimming and flying animals is dictated by fluid drag," Proceedings of the National Academy of Sciences, vol. 115, no. 32, pp. 8116-8118, 2018.

[2] J. Parmentier, S. Lejeune, M. Maréchal et al., "A drop does not fall in a straight line: a rationale for the width of stalagmites," Proceedings of the Royal Society A: Mathematical, Physical and Engineering Sciences, vol. 475, no. 2231, Article ID 20190556, 2019.

[3] P. K. Wang and C.-C. Chueh, "A numerical study on the ventilation coefficients of falling lobed hailstones," Atmospheric Research, vol. 234, Article ID 104737, 2020.

[4] A. G. Churbanov, O. Iliev, V. F. Strizhov, and P. N. Vabishchevich, "Numerical simulation of oxidation processes in a cross-flow around tube bundles," Applied Mathematical Modelling, vol. 59, pp. 251-271, 2018.

[5] J. Lai, L. Sun, J. Yang et al., "Experimental and numerical analyses on flow-induced vibration of a nuclear engineering test reactor internal," Nuclear Engineering and Design, vol. 340, pp. 335-346, 2018.
[6] A. M. Rashad, W. A. Khan, S. M. M. EL-Kabeir, and A. M. A. EL-Hakiem, "Mixed convective flow of micropolar nanofluid across a horizontal cylinder in the saturated porous medium," Applied Sciences, vol. 9, no. 23, Article ID 5241, 2019.

[7] Z. Duan, B. He, and Y. Duan, "Sphere drag and heat transfer," Scientific Reports, vol. 5, no. 1, pp. 12304-12307, 2015.

[8] Y. Li, Z. Li, C. Wang, M. Qi, and W. Ren, "Particle image velocimetry analysis of plane flow field induced by drill string planetary motion based on superposition principle," Journal of Petroleum Science and Engineering, vol. 170, pp. 121-129, 2018.

[9] J. Yao, W. Lou, G. Shen, Y. Guo, and Y. Xing, "Influence of inflow turbulence on the flow characteristics around a circular cylinder," Applied Sciences, vol. 9, no. 17, Article ID 3595, 2019.

[10] W. He, J. M. Pérez, P. Yu, and L. K. B. Li, "Non-modal stability analysis of low-Re separated flow around a NACA 4415 airfoil in ground effect," Aerospace Science and Technology, vol. 92, pp. 269-279, 2019.

[11] Y. Fan, Y. Bao, C. Ling, Y. Chu, X. Tan, and S. Yang, "Experimental study on the thermal management performance of air cooling for high energy density cylindrical lithium-ion batteries," Applied Thermal Engineering, vol. 155, pp. 96-109, 2019.

[12] H. Liang and R.-Q. Duan, "Effect of lateral end plates on flow crossing a yawed circular cylinder," Applied Sciences, vol. 9, no. 8, Article ID 1590, 2019.

[13] A. Chakravarty, "Numerical analysis of fluid flow and heat transfer in 2D sinusoidal wavy channel," in Proceedings of the Student's conference Czech Technical University, Prague, Zcech Republic, April 2017.

[14] O. Veli, N. Tokgoz, and B. Sahin, "Flow characteristics and heat transfer enhancement in 2D corrugated channels," "International Journal of Mechanical, Industrial and Aerospace Science", vol. 7, pp. 2084-2088, 2013.

[15] B. Hoareau, M. Gradeck, and M. Lebouché, "Local analysis of heat transfer inside corrugated channel," International Journal of Heat and Mass Transfer, vol. 48, pp. 1909-1915, 2005.

[16] N. Ali, M. Sajid, Z. Abbas, and T. Javed, "Non-Newtonian fluid flow induced by peristaltic waves in a curved channel," European Journal of Mechanics - B: Fluids, vol. 29, no. 5, pp. 387-394, 2010.

[17] G. Bizzarri, V. Di Federico, and S. Cintoli, "Stokes flow between sinusoidal walls," WIT Transactions on Engineering Sciences, vol. 36, pp. 323-332, 2002.

[18] J. W. Elder, "Steady flow through non-uniform gauzes of arbitrary shape," Journal of Fluid Mechanics, vol. 5, no. 3, pp. 355-368, 1959.

[19] A. A. Memon, H.-u. Shaikh, and M. A. Memon, "Finite element's analysis of fluid flow through the rectangular channel with inclined screens settled at angles," in Proceedings of the 2nd International Conference on Computing Mathematics and Engineering Technologies (iCoMET), pp. 1-6, Sukkur IBA University, Sukkur, Pakistan, January 2019.

[20] A. A. Memon, "Analysis of optimum velocity and pressure of the air flow through the screens with the help of Resistance coefficient," Sukkur IBA Journal of Computing and Mathematical Sciences, vol. 3, pp. 51-57, 2019.

[21] G. Hauke and T. J. R. Hughes, "A unified approach to compressible and incompressible flows," Computer Methods in Applied Mechanics and Engineering, vol. 113, no. 3-4, pp. 389-395, 1994. 
[22] I. Khan, A. A. Memon, M. A. Memon et al., "Finite element least square technique for Newtonian fluid flow through a semicircular cylinder of recirculating region via COMSOL multiphysics," Journal of Mathematics, vol. 2020, 2020.

[23] A. A. Memon, H.-u. Shaikh, M. A. Soomro, A. G. Shaikh, and A. H. Shaikh, "Modeling and simulation of Newtonian fluid flow through two-dimensional backward-facing step channel with finite element's technique," Indian Journal of science and technology, vol. 12, no. 32, pp. 1-6, 2019.

[24] A. A. Memon, H. Alotaibi, M. A. Memon et al., "Finite element analysis of fluid flow through the screen embedded between parallel plates with high Reynolds numbers," Journal of Function Spaces, vol. 2021, Article ID 6695733, 2021.

[25] A. A. Memon, M. A. Memon, K. Bhatti, and G. M. Shaikh, "Finite element simulation of Newtonian and non-Newtonian fluid through the parallel plates affixed with single screen," European Journal of Pure and Applied Mathematics, vol. 13, no. 1, pp. 69-83, 2020. 